\title{
Intra-articular use of hyaluronic acid in the treatment of osteoarthritis
}

\author{
Alberto Migliore ${ }^{1,2}$ \\ Mauro Granata ${ }^{3}$ \\ 'UOS of Rheumatology S. Pietro \\ Fatebenefratelli Hospital, Rome, Italy; \\ ${ }^{2} \mathrm{AFaR}$ Research Center, S. Pietro, \\ Rome, Italy; ${ }^{3}$ UOS of Rheumatology \\ S. Filippo Neri Hospital, Rome, Italy
}

\begin{abstract}
Osteoarthritis is one of the leading causes of disability in the elderly. The changes in the lubricating properties of synovial fluid lead to significant pain and loss of function. More than ten years have passed from the first studies. Up till now many authors have supported intra-articular hyaluronan (HA) therapy as not only a symptom-modifying therapy but also a treatment which may significantly decrease the rate of deterioration of joint structure. In this review we report data relative to knee and hip treatment. The ongoing studies continue to further our understanding of the fundamental mechanisms that likely underlie the therapeutic benefits of this treatment but, despite recent progress, many unresolved issues require further study. Large scale double blind controlled studies must be carried out to confirm these promising data and produce meaningful guidelines.
\end{abstract}

Keywords: osteoarthritis, hyaluronan, intra-articular injections, ultrasound-guided, viscosupplementation

\section{Introduction}

Osteoarthritis is one of the leading causes of disability in the elderly. The changes in the lubricating properties of synovial fluid lead to significant pain and loss of function. Viscosupplementation, the injection of hyaluronic acid (HA) into the joints, improves the biochemical properties of synovial fluid into osteoarthritis joints. The clinical effect is pain relief and disease modifying activity (Balazs and Denlinger 1993; Pelletier and Martel-Pelletier 1993).

Although there are many nonsurgical therapies available for the treatment of pain associated with osteoarthritis (OA), their long-term use and safety have not been systematically followed. Intra-articular (IA) hyaluronan therapy has been used in the treatment of symptoms associated with OA of the knee with a very favorable safety profile (Pagnano and Westrich 2005). In this connection, the physiological and pharmacological characteristics of HA have recently been reviewed. The high concentration of HA in spinal fluid (SF) is essential for normal joint function. The altered properties of the synovial fluid in OA, the decrease in molecular size and concentration of HA leads to reduced rheologic properties, which affect the lubrication and protection mechanism of action of IA hyaluronan and is probably more complex than previously thought (Maneiro et al 2004).

The hypothesis that HA could bind to specific receptors is now widely accepted. The cluster determinant 44 (CD44), the intracellular adhesion molecule-1 (ICAM-1) and the receptor for hyaluronate-mediated motility (RHAMM) expressed in many cells, are some of those implicated in the process (Hodge-Dufour et al 1997; Siegelman et al 1999). The binding of HA with its specific receptors has been reported to trigger various intracellular signal events such as cytokine release and stimulation of cell cycle proteins. The consequences of these interactions is to stimulate cell functional activities such as cell migration and proliferation (Cao et al 2004). 
More than ten years have passed since the original explanation by Balazs and Denlinger (1993) that the favorable effects of IA HA therapy in OA was related only to the restoration of SF rheological properties (Goldberg and Buckwalter 2005). Many subsequent studies have supported that IA HA therapy is a symptom-modifying approach that has been found to be safe and effective for reducing pain due to OA of the knee and furthermore may also significantly decrease the rate of deterioration of joint structure. This statement stems from different evidences. First of all, the complex biochemical effects of HAs in the synovium and extracellular matrix of the articular cartilage, including interactions between exogenously administered HA and articular cartilage, subchondral bone, matrix proteoglycans, and collagens. In the second place, the effects of HA administration in animal models of OA, including total or partial meniscectomy and anterior cruciate ligament transectomy. Thirdly, the results of clinical trials using one HA, Hyalgan (sodium hyaluronate, molecular weight 500-730 kDa) that evaluated structural outcomes, such as joint-space width, chondrocyte density and vitality, and arthroscopic evaluation of chondropathy. Localized severe acute inflammatory reactions reported with repeated treatment in some patients are not a class effect but may be linked to physicochemical characteristics of hylanbased treatment (Arrich et al 2005; Pagnano and Westrich 2005; Goldberg and Buckwalter 2005).

Studies on the effects of HAs of different molecular weight (MW) on specific receptors have shown that the results can vary depending on the size of the HA molecules used. For example low MH HA fragments generated during joint inflammation may promote chemokine gene expression by macrophages. Ohkawara and colleagues (2000) noticed that HA preparation of MW $0.2 \times 106$ Da was more effective in maintaining the survaival of blood eosinophils than HA with an MW of $3 \times 106-6 \times 106 \mathrm{Da}$, proposing that the effect derived from the increased expression of granulocyte macrophage colony-stimulating factor after CD44 activation (Ohkawara et al 2000).

\section{Different HA products}

Presently, there are several different HA products available for injection. However, the source of hyaluronic acid varies. Some products are extracted from rooster combs but a contraindication to treatment would be an allergy to eggs or chicken.

Hyaluronic acid is available in a variety of products with different molecular weight ranging from $500 \mathrm{KD}$ to more than 90 millions D (Table 1). Hylans are cross-linked hyaluronic acids, which gives them a higher molecular weight and increased elastoviscous properties.

\section{HA products in the intra-articular treatment of knee OA}

The first studies on the use of HA in human knee OA were carried out in the 1970s by Rydell and Balasz (1971) and Peyron and Balasz (1974). In 1974, Peyron injected 1, 2, or $3 \mathrm{ml}$ of HA (Healon) in 23 knees and obtained positive responses in pain and function in $74 \%$ of patients. The treatment with two injections of $2 \mathrm{ml} \mathrm{HA}$ each appeared to have the best outcome.

Recent papers have reviewed clinical trials published in the last years using different HA preparation and the results of these trials are summarized in Table 2.

Data support the benefit and safety of repeated treatment with IA HA. Many trials indicate that sodium hyaluronate (molecular weight [MW] 500-730 kDa) is well tolerated and as effective after multiple courses of treatment as it is after a single course (Pagnano and Westrich 2005). Other data state that viscosupplementation is an effective treatment for patients with knee OA who have ongoing pain or are unable to tolerate conservative treatment or joint replacement.

Viscosupplementation appears to have a slower onset of action than intra-articular steroids, but the effect seems to last longer (Wang et al 2004).

HA not only relieves the symptoms of OA but also modifies the structure of the diseased joint and the rate of

Table I Molecular weights of hyaluronic acid (HA) products

\begin{tabular}{llll}
\hline Trade name & Molecular weight & Source & Mg/ml \\
\hline Hyalgan & $500-730 \mathrm{KDa}$ & HA extracted from rooster combs & $20 \mathrm{mg} / 2 \mathrm{ml}$ \\
Hyalubrix $\gtrless$ & $1500 \mathrm{KDa}$ & Fermentative HA & $30 \mathrm{mg} / 2 \mathrm{ml}$ \\
Artz (Supartz) & $800-1170 \mathrm{KDa}$ & HA extracted from rooster combs & $25 \mathrm{mg} / 2.5 \mathrm{ml}$ \\
Synovial (Jointex) & $800-1200 \mathrm{KDa} \mathrm{HA}$ & Fermentative HA & $16 \mathrm{mg} / \mathrm{ml}$ \\
Synvisc & $6000 \mathrm{KDa}$ & HA extracted from rooster combs & $16 \mathrm{mg} / 2 \mathrm{ml}$ \\
Durolane & $90 \mathrm{millions} \mathrm{Da}$ & Synthetic HA & $20 \mathrm{mg} / 3 \mathrm{ml}$ \\
Euflexxa & $2.400-3.600 \mathrm{kDa}$ & Fermentative HA & $20 \mathrm{mg} / 2 \mathrm{ml}$ \\
\hline
\end{tabular}


Table 2 Recent clinical trials using HA

\begin{tabular}{|c|c|c|c|}
\hline Author / Year & $\mathbf{N}$ trials & Intervention & Conclusions \\
\hline Lo et al 2004 & 22 selected trials & $\begin{array}{l}\text { Hyalgan, Synvisc, Artzal, Suplasyn, } \\
\text { BioHy, Orthovisc }\end{array}$ & $\begin{array}{l}\text { Measurable effect when compared with } \\
\text { placebo. Highest-molecular-weight HAs } \\
\text { may be more. Heterogeneity of these } \\
\text { studies limits definitive conclusions. }\end{array}$ \\
\hline Aggarwal et al 2004 & Five case series I 3 RCTs & Synvisc low-molecular-weight HA & $\begin{array}{l}\text { High-molecular-weight HAs effective. } \\
\text { Conflicting results with low-molecular- } \\
\text { weight. Slower onset of action than } \\
\text { intra-articular steroids, but the effect last } \\
\text { longer. }\end{array}$ \\
\hline Wang et al 2004 & $\begin{array}{l}\text { Twenty blinded randomized } \\
\text { controlled trials }\end{array}$ & Hyaluronic acid versus placebo & Therapeutic efficacy and safety \\
\hline Axe et al 2005 & $\begin{array}{l}\text { Potential use in the treatment of } \\
\text { inflammatory arthropathies (eg, } \\
\text { acute joint trauma and fractures) }\end{array}$ & Hyaluronans & $\begin{array}{l}\text { Trials limited in design and patient } \\
\text { number. Moderate results. Controlled } \\
\text { clinical trials are warranted. }\end{array}$ \\
\hline Goldberg et al 2005 & $\begin{array}{l}\text { A review on a Medline search } \\
\text { through June } 2004\end{array}$ & Hyalgan, Supartz, Synvisc & $\begin{array}{l}\text { In addition to relieving the symptoms, } \\
\text { HAs also modify the structure of the } \\
\text { diseased joint and the rate of OA disease } \\
\text { progression. }\end{array}$ \\
\hline Arrich et al 2005 & Twenty-two trials & Hyaluronic acid & $\begin{array}{l}\text { The data available did not allow an } \\
\text { appropriate assessment of any end point. } \\
\text { Adverse events occurred slightly more } \\
\text { often. }\end{array}$ \\
\hline Bellamy et al 2005 & $\begin{array}{l}\text { Thirty-seven trials included } \\
\text { comparisons of hyaluronan/hylan } \\
\text { and placebo, nine trials included } \\
\text { comparisons of intra-articular } \\
\text { corticosteroids, and five trials } \\
\text { included comparisons of (NSAIDs) }\end{array}$ & $\begin{array}{l}\text { Adant, Arthrum H, Artz (Artzal, } \\
\text { Supartz), BioHy (Arthrease), Duro- } \\
\text { lane, Fermathron, Go-on, Hyalgan, } \\
\text { Hylan G-F } 20 \text { (Synvisc Hylan G-F } \\
\text { 20), NRD- IOI, Orthovisc, Ostenil, } \\
\text { Replasyn, SLM- I0, Suplasyn, Synject } \\
\text { and Zeel compositum }\end{array}$ & $\begin{array}{l}\text { The effects against 'placebo' controls } \\
\text { generally supported the efficacy. Delayed } \\
\text { effects on pain and function. Comparable } \\
\text { efficacy against NSAIDs and longer-term } \\
\text { benefits against IA corticosteroids. Few } \\
\text { adverse events were reported. }\end{array}$ \\
\hline Pagnano et al 2005 & $\begin{array}{l}\text { Databases were searched using } \\
\text { the terms hyaluronan, sodium } \\
\text { hyaluronate, hyaluronic acid, hylan, } \\
\text { hylan G-F } 20\end{array}$ & $\begin{array}{l}\text { Hyaluronan, sodium hyaluronate, } \\
\text { hyaluronic acid, hylan, hylan G-F } 20\end{array}$ & $\begin{array}{l}\text { Hyaluronans are safe and effective in the } \\
\text { treatment of pain. Sodium hyaluronate } \\
\text { decrease the rate of deterioration of joint } \\
\text { structure }\end{array}$ \\
\hline
\end{tabular}

Abbreviations: IA, intra articular; RCT, randomized controlled trials; HA, hyaluronic acid; OA, osteoarthritis.

OA disease progression, at least early in the evolution of the disease process (Ohkawara et al 2000).

Based on the aforementioned analyses, viscosupplementation is an effective treatment for OA of the knee with beneficial effects on pain, function and patient global assessment at different post injection periods but especially at the 5 to 13 week post injection period with a dramatic effect on the reduction of NSAIDs monthly consumption.

However, there are few randomized head-to-head comparisons of different viscosupplements and readers should be cautious, therefore, in drawing conclusions regarding the relative value of different products.

In some analyses, viscosupplements were comparable in efficacy to systemic forms of treatment, with more local reactions but fewer systemic adverse events. In other analyses HA products had more prolonged effects than IA corticosteroids. Overall, the aforementioned analyses support the use of the
HA class of products in the treatment of knee OA (Lo et al 2003). In one systematic review, IA HA has not been proven clinically effective and may be associated with minimal and transient risk of adverse events (Arrich et al 2005).

At present no case of septic arthritis has been reported in any clinical trial. Most adverse events are minor and transient at the injection site. Painful post-injection reaction occurred in $1 \%-2 \%$ of patients, the pain did not last more than 72 hours. Systemic allergic reactions due to individual hypersensitivity were rarely recorded. Therefore viscosupplementation seems to be an efficacious and safe treatment.

The heterogeneity of these studies limits definitive conclusions on different treatment regimen with diverse molecular weight HA but in our opinion we think that the different HA products act with the same class effect (Axe and Shields 2005). 
Furthermore preliminary data indicate a potential use for hyaluronans in the treatment of inflammatory arthropathies (eg, acute joint trauma and fractures) that require long periods of immobilization, and in tissue engineering for chondral defects. Although the trials that have investigated the use of hyaluronan therapy for the management of traumatic and degenerative musculoskeletal disorders seen in sports medicine have limitations in design and patient number, the results have been promising and suggest that larger controlled clinical trials are warranted (Peyron and Balasz 1974).

\section{HA products in the intra-articular treatment of hip OA}

Few data exists in literature about the viscosupplementation of hip OA in spite of it is the second most common site of the disease after the knee (Pelletier and Martel-Pelletier 1993; Pagnano and Westrich 2005). One of the limiting factors for this practice might be related to the injection procedure. The intra-articular injection of the hip is not as easy as for the knee, mainly for anatomical features of the joint and the proximity of "sensitive" structures such as the femoral artery and nerves. Even though hip injection may be performed "blindly", failure rate is nevertheless significant (Jones et al 1993 ) and when using a slow absorbing viscosupplement like hyaluronan, the potential local complications may jeopardize the therapeutic benefit (Jackson et al 2002). For such reasons, it has been suggested to perform intra-articular injection of the hip under radiological or ultrasound control. Although several ultrasound guidance techniques were available (Qvistgaard et al 2001), we developed a personal one using an antero-superior approach (Migliore et al 2003).

Since no single or double blind randomized controlled trials have yet been published, we analyzed all the available trials on MEDLINE to assess viscosupplementation for the treatment of hip OA (Table 3).
The efficacy data underlines significant improvement in pain and function as has been shown in the knee OA studies despite the small size of the sample. The short period of observation has not seen a change in the natural history of the disease. In order to confirm these promising data, large scale double blind controlled studies must be carried out.

\section{Conclusions}

The ongoing studies continue to further our understanding of the fundamental mechanisms that likely underlie the therapeutic benefits of this treatment. The meta-analysis in progress are further establishing a role for viscosupplementation in ameliorating the symptoms of knee and hip osteoarthritis. At the moment it is clear that viscosupplementation is more efficacious in the initial and intermediate stages of OA more than at an advanced stages and that this therapy is exceptionally safe compared with other OA treatments.

Despite recent progress, many unresolved issues require further study. These include determining the costeffectiveness of viscosupplementation, exploring the therapeutic effectiveness and safety, clearly defining the relation between molecular weight and the clinical effectiveness of the various hyaluronan products, establishing optimal dosing regiments, evaluating efficacy in different OA populations, determining how to best incorporate viscosupplementation into the evolving OA treatments algorithms, exploring the potential for treatment of OA joints other than the knee, as already shown for the hip, and assessing potential synergistic or additive effects with other modalities such NSAIDs and SYSADOA.

It is encouraging that hyaluronan-based therapy is proving to be safe and effective for many patients with symptomatic hip and knee OA despite the fact that many unresolved issues requiring further study remain. If hyaluronan derivatives are eventually proven to have clear

Table $3 \mathrm{~V}$ scosupplementation studies for the treatment of hip osteoarthritis

\begin{tabular}{llllll}
\hline Author year & HA & Nr of pts. & Outcome measures & Months of follow-up & Nr.of injections \\
\hline Brigantini 1994 & Hyalgan & 44 & P/GA/NSAIDs & 6 & $3-5$ \\
Brocq 2002 & Synvisc & 22 & LI & $1-6$ & $1-2$ \\
Conrozier 2003 & Synvisc & 57 & P/W/GA & $3-6$ & $1-2$ \\
Vad 2003 & Synvisc & 22 & P/AAOS/LLCSS & 12 & 3 \\
Migliore 2003 & Hyalgan & 28 & P/LI/NSAIDs & NG & $1-3$ \\
Caglar-Yagci 2005 & Synvisc & 14 & P/LI/I5 mWT & 3 & 3 \\
Berg 2004 & Durolane & 31 & W/GA & 3 & 1 \\
Migliore 2005 & Synvisc & 26 & P/LI/NSAIDs & 12 & $1-2$ \\
Tikiz 2005 & Synvisc vs Ostenil & 25 vs I8 & P/W/LI & 6 & 3 \\
Migliore 2006 & Synvisc & 36 & P/W/NSAIDs & 18 & $\mathrm{I}$ \\
\hline A 200
\end{tabular}

Abbreviations: P, pain; GA, global assessment; 15 mWT, I5-meter walking time; NSAIDs, NSAIDs consumption; LI, Lequesne index; W, Womac index; AAOS, American Academy of Orthopaedic Surgeons; LLCSS, Lower Limb Core Scale Score. 
disease-modifying effects, it may then be reasonable to consider their use early in cartilage injury or disease, before the onset of syntomatic OA.

Additional well-designed randomized controlled trials with high methodological quality are needed to resolve the continued uncertainty about the therapeutic effects of different types of hyaluronic acid products on osteoarthritis in various clinical situations and patient populations (Wang et al 2004).

At the moment the Arthritis Foundation believes that while more studies are needed to determine the long-term results of such treatments, HA provides an option for people with knee OA who have not responded well to drug treatments or exercise and physical therapy. It also may be appropriate for those who may be at increased risk for upper gastrointestinal (GI) complications, such as patients who: are over 65 years old; are taking oral corticosteroids and/or anticoagulants; have a history of peptic ulcer disease or upper GI bleeding; are smokers; and consume high volumes of alcohol. HA also may be considered in patients with kidney failure.

\section{Disclosure}

The authors report no conflicts of interest.

\section{References}

Aggarwal A, Sempowski IP. 2004. Hyaluronic acid injections for knee osteoarthritis. Systematic review of the literature. Can Fam Physician, 50:249-56.

Arrich J, Piribauer F, Mad P, et al. 2005. Intra-articular hyaluronic acid for the treatment of osteoarthritis of the knee: systematic review and meta-analysis. CMAJ, 172:1039-43.

Axe MJ, Shields CL Jr. 2005. Potential applications of hyaluronans in orthopaedics: degenerative joint disease, surgical recovery, trauma and sports injuries. Sports Med, 35:853-64.

Balazs EA, Denlinger J. 1993. Viscosupplementation: A new concept in the treatment of osteoarthrtitis. J Rheumatol, 20(Suppl 39):3-9.

Bellamy N, Campbell J, Robinson V, et al. 2005. Viscosupplementation for the treatment of osteoarthritis of the knee. Cochrane Database Syst Rev, 2:CD005321.

Berg P, Olsson U. 2004. Intra-articular injection of non animal stabilized hyaluronic acid for osteoarthritis of the hip: a pilot study. Clin Exp Rheumatol, 22:300-6.

Brigantini A, Molinari F. 1994. A pilot clinical evalutation of the treatment of hip osteoarthritis with hyaluronic acid. Curr Ther Res, 55:319-30.

Brocq Q, Tran G, Breueil V, et al. 2002. Hip osteoarthritis: Short term efficacy of viscosupplementation by hylan GF-20. An open-label study in 22 patients. Joint Bone Spine, 69:388-91.

Caglar-Yagci H, Unsal S, Yagci I, et al. 2005. Safety and efficacy of ultrasound-guided hylan G-F 20 injection in osteoarthritis of the hip: a pilot study. Rheumatol Int, 25:341-4.

Cao JJ, Singleton PA, Majumdar S, et al. 2005. Hyaluronan increases RANKL expression in bone marrow stromal cells through CD44. J Bone Miner Res, 20:30-40.
Conrozier T, Bertin P, Mathieu P, et al. 2003. Intra-articular injection of hylan GF-20 in patients with symptomatic hip osteoarthritis: an openlabel, multi-centre pilot study. Clin Exp Rheumatol, 21:605-10.

Goldberg VM, Buckwalter JA. 2005. Hyaluronans in the treatment of osteoarthritis of the knee: evidence for disease-modifying activity. Osteoarthritis Cartilage, 13:216-24.

Hodge-Dufour J, Noble PW, Horton MR, et al. 1997. Induction of IL-12 and chemokines by hyaluronan requires adhesion-dependent priming of resident but not elicited macrophages. J Immunol, 159:2492-500.

Jackson DW, Evans NA, Thomas BM. 2002. Accuracy of needle placement into the intra-articular space of the knee. J Bone Joint Surg Am, 84A:1522-1527.

Jones A, Regan M, Ledingham J, et al. 1993. Importance of placement of intra-articular steroid injections. BMJ, 307:1329-30.

Lo GH, LaValley M, McAlindon T, et al. 2003. Intra-articular hyaluronic acid in treatment of knee osteoarthritis: a meta-analysis. JAMA, 290:3115-21.

Maneiro E, de Andres MC, Fernandez-Sueiro JL, et al. 2004. The biological action of hyaluronan on human osteoarthritic articular chondrocites. The importance of molecular weight. Clin Exp Rheumatol, 22:307-12.

Migliore A, Martin LS, Alimonti A, et al. 2003. Efficacy and safety of viscosupplementation by ultrasound guided intra-articular injection in osteoarthritis of the hip. Osteoarthritis Cartilage, 11:305-6.

Migliore A, Tormenta S, Valente C, et al. 2005. Intra-articular treatment with Hylan G-F 20 under ultrasound guidance in hip osteoarthritis. Clinical results after 12 months follow-up. Reumatismo, 57:36-43.

Migliore A, Tormenta S, Martin Martin LS, et al. 2006. The symptomatic effects of intra-articular administration of hylan G-F 20 on osteoarthritis of the hip: clinical data of 6 months follow-up. Clin Rheumatol, 25:389-93.

Ohkawara Y, Tamura G, Iwasaki T, et al. 2000. Activation and transforming growth factor beta production in eosiniphils by hyaluronan. $\mathrm{Am}$ J Respir Cell Mol Biol, 23:444-51.

Pagnano M, Westrich G. 2005. Successful nonoperative management of chronic osteoarthritis pain of the knee: safety and efficacy of retreatment with intra-articular hyaluronans. Osteoarthritis Cartilage, 13:751-61.

Pelletier JP, Martel-Pelletier J. 1993. The pathophysiology of osteoarthritis and the implication of the use of hyaluronan and hylan as therapeutic agents in viscosupplementation. J Rheumatol (Suppl 39):19-24.

Peyron JG, Balasz EA. 1974. Preliminary clinical assessment of Na-hyaluronate injection into human arthritic joint. Pathol Biol (Paris), 22:731-6.

Qvistgaard E, Kristoffersen H, Terslev L, et al. 2001. Guidance by ultrasound of intra-articular injections in the knee and hip joints. Osteoarthritis Cartilage, 9:512-17.

Rydell NW, Balasz EA. 1971. Effect of intra-articular injection of hyaluronic acid on the clinical symptoms of osteoarthritis and on granulation tissue formation. Clin Orthop, 80:25-32.

Siegelman MH, DeGrendele HC, Estess P. 1999. Activation and interaction of CD44 and hyaluronan in immunological system. J Leukoc Biol, 66:315-21.

Tikiz C, Unlu Z, Sener A, et al. 2005. Comparison of the efficacy of lower and higher molecular weight viscosupplementation in the treatment of hip osteoarthritis. Clin Rheumatol, 24:244-50.

Vad VB, Sakalkale D, Sculco TP, et al. 2003. Role of hylan G-F 20 in treatment of osteoarthritis of the hip joint. Arch Phys Med Rehabil, 84:1224-6.

Wang CT, Lin J, Chang CJ, et al. 2004. Therapeutic effects of hyaluronic acid on osteoarthritis of the knee. A meta-analysis of randomized controlled trials. J Bone Joint Surg Am, 86-A:538-45. 
